\title{
$\mathrm{M}|\mathrm{R}| \mathrm{S}$ Internet Journal Nitride Semiconductor Research
}

\section{UV-Specific (320-365 nm) Digital Camera Based On a 128x128 Focal Plane Array of GaN/AIGaN p-i-n Photodiodes}

\author{
J.D. Brown ${ }^{1}$, J. Boney ${ }^{1}$, J. Matthews ${ }^{1}$, P. Srinivasan ${ }^{1}$, J.F. Schetzina ${ }^{1}$, Thomas Nohava ${ }^{2}$, Wei Yang ${ }^{2}$ \\ and Subash Krishnankutty ${ }^{2}$ \\ ${ }^{1}$ North Carolina State University, \\ ${ }^{2}$ Honeywell Technology Center,
}

(Received Wednesday, June 14, 2000; accepted Thursday, July 13, 2000)

\begin{abstract}
An ultraviolet-specific (320-365 nm) digital camera based on a 128x128 array of backsideilluminated GaN/AlGaN p-i-n photodiodes has been successfully developed. The diode structure consists of a base n-type layer of $\mathrm{AlGaN}(\sim 23 \% \mathrm{Al})$ followed by undoped and then p-type GaN layers deposited by metal organic vapor phase epitaxy. Double-side polished sapphire wafers serve as transparent substrates. Standard photolithographic, etching, and metallization procedures were employed to fabricate the devices. The fully-processed photodiode array was hybridized to a silicon readout integrated circuit (ROIC) using In bump bonds for electrical contact. The UV camera was operated at room temperature at frame rates ranging from 15 to $240 \mathrm{~Hz}$. A variety of UV scenes were successfully recorded with this configuration.
\end{abstract}

\section{Introduction}

The III-V nitride semiconductors have received much attention in recent years. These materials, which have large direct bandgaps, are ideal for optoelectronic applications in the blue, green, and ultraviolet (UV) regions of the electromagnetic spectrum. Light emitting diodes and laser diodes based on these materials have been developed and commercialized [1] [2] [3] [4] [5] [6] [7] [8] [9] [10]. In addition, UV photodetectors based on photoconductors and junction detectors have been reported [11] [12] [13]. In addition, UV imaging arrays have recently been demonstrated based on photoconducting detectors [14] and on 32x32 arrays of heterostructure p-i-n photodiodes [15] [16]

This paper reports the successful development of a larger-format imaging array consisting of $128 \times 128$ GaN/AlGaN p-i-n photodiodes designed to sense radiation in the 320-365 nm portion of the UV spectral region. First, the experimental procedures employed to synthesize, process, and characterize discrete photodiodes and photodiode arrays are described. Then, experimental results obtained using this new large-format UV imager are presented including a selection of UV images.

\section{Experimental Details}

\subsection{Photodiode Structure}

Diode structures were prepared by MOVPE both at North Carolina State University (NCSU) and at the Honeywell Technology Center (HTC) using low-pressure, vertical-flow reactors that employ high speed substrate rotation during film growth. The photodiode structures were deposited onto 2 inch diameter c-plane sapphire substrates. A thin AlN buffer layer was deposited at $500-650{ }^{\circ} \mathrm{C}$ to initiate growth; all subsequent layers were grown at $1050-1080^{\circ} \mathrm{C}$.

A schematic of the photodiode structure employed in the present work is shown in Figure 1. The thin low temperature AlN nucleation layer is first deposited followed by a $\sim 1.0 \mu \mathrm{m}$ thick n-type silicon doped $\mathrm{Al}_{0.23} \mathrm{Ga}_{0.77} \mathrm{~N}$ base layer. An undoped $0.2 \mu \mathrm{m}$ thick GaN layer is then deposited followed by a $0.5 \mu \mathrm{m}$ thick p-type magnesium doped $\mathrm{GaN}$ layer to complete the p-i$\mathrm{n}$ heterostructure. Note that this device structure is designed for through-the-substrate illumination. The photodiode structure shown in Figure 1 responds to UV light in the wavelength band from about $320 \mathrm{~nm}$ to 365 $\mathrm{nm}$. At wavelengths shorter than $320 \mathrm{~nm}$, the incoming light is absorbed in the thick AlGaN base layer ( 23\% $\mathrm{Al})$ and the junction is not illuminated. Likewise, the diode does not respond to wavelengths greater than 365 
$\mathrm{nm}$, since this corresponds to the optical absorption edge of $\mathrm{GaN}$ at $300 \mathrm{~K}$.

Using the basic device structure shown in Figure 1, it is possible to tailor the responsivity window of the UV detector by adjusting the aluminum mole fraction present in each of the three layers. Thus, it is possible to synthesize detectors that sense different UV "colors".

\subsection{Device Processing of Photodiode Arrays}

All device processing was completed at NCSU using standard semiconductor processing techniques which included photolithography using appropriately-designed masks, reactive ion etching to define mesa structures, and metallizations to provide ohmic contacts to the ntype and p-type layers of the device. The $128 \times 128$ photodiode array consists of 16,384 mesa diodes. The mesas are $32 \mu \mathrm{m}$ squares on a $38 \mu \mathrm{m}$ pitch, corresponding to a fill factor of $71 \%$. Photolithographically defined $\mathrm{Ni}$ was used to define the mesas, and $\mathrm{BCl}_{3}$ reactive ion etching was used to etch the mesas down to the n-type AlGaN base layer. $\mathrm{Ni} / \mathrm{Au}$ and Ti/Al metallizations, followed by an anneal at $600-700{ }^{\circ} \mathrm{C}$, were employed to obtain p-type and n-type ohmic contacts, respectively.

The $128 \times 128$ photodiode array was hybridized to a silicon readout integrated circuit (ROIC) using In bump bonds. In this procedure, photoresist was used to define $18 \mu \mathrm{m}$ circular vias at all of the p-contact and $\mathrm{n}$-contact points of the photodiode array, and at the corresponding metal pads of the Si ROIC. A dedicated UHV deposition system which employs MBE-type effusion cells was then used to precisely deposit a uniform array of In bumps of thickness $5.0 \mu \mathrm{m}$ onto both the photodiode array and the Si ROIC (see Figure 2). The photodiode array was then hybridized to the Si ROIC using a RD Automation model M8-G flip chip aligner bonder. The $128 \times 128$ hybridized focal plane array (FPA) was then cemented onto an 84-pin leadless chip carrier (LCC). Outputs from the ROIC were then wire bonded to the LCC using gold wires as shown in Figure 3. The UV camera chip was then inserted into a matching 84 pin socket located on a circuit board in the UV camera as shown in Figure 4. The UV camera, shown in Figure 5, employs a fused quartz focusing lens along with hardware and software for reading out, displaying, and storing UV images from the 128x128 FPA at selected frame rates under computer control.

Alphanumeric and geometric objects were recorded with the $128 \times 128 \mathrm{UV}$ digital camera by using templates that were back-illuminated with an UV lamp [15]. UV images of an oxy acetylene torch and a TIG arc welder were also obtained.

\section{Results and Discussion}

Several large $(200 \mu \mathrm{m} \times 200 \mu \mathrm{m})$ discrete mesa photodiodes were processed from the same wafers as the $128 \times 128$ photodiode arrays so that responsivity and detectivity measurements [15] could be conducted. The spectral responsivity obtained for one of these representative photodiodes is shown in Figure 6. It is seen from the figure that the responsivity begins to increase rapidly beginning at about $365 \mathrm{~nm}$ and reaches a peak value of $0.21 \mathrm{~A} / \mathrm{W}$ at $358 \mathrm{~nm}$, corresponding to an internal quantum efficiency [15] of $82 \%$. The responsivity remains fairly constant until about $320 \mathrm{~nm}$ after which it decreases rapidly. Thus, the nitride photodiode, and the corresponding 128x128 FPA, is visible-blind (but not solar-blind) and responds only to a band of UV wavelengths in the range $320-365 \mathrm{~nm}$.

The 300K dynamic resistance of the photodiode test structure at zero-bias $\mathrm{R}_{0}$ was measured using a special low-noise shielded enclosure and shielded probe tips. These measurements were combined with the device area $\mathrm{A}$ to obtain the $\mathrm{R}_{0} \mathrm{~A}$ product, a well known figure of merit for photodiode detectors. The $\mathrm{R}_{0} \mathrm{~A}$ product was then used to obtain an estimate of the detector detectivity D*, a measure of the device sensitivity [15]. From such a procedure, a $300 \mathrm{~K}$ value of $\mathrm{D}^{*}=6.3 \times 10^{13} \mathrm{~cm}$ $\mathrm{Hz}^{1 / 2} \mathrm{~W}^{-1}$ at $358 \mathrm{~nm}$ was obtained for several UV p-i-n photodiode test structures. This is one of the largest $\mathrm{D}^{*}$ values ever obtained for any semiconductor photodiode at any temperature.

In addition, selected $200 \mu \mathrm{m} \times 200 \mu \mathrm{m}$ photodiodes were subjected to light pulses from a xenon lamp and their transient response was measured using a fast oscilloscope. From these measurements we estimate that the rise time of the nitride photodiodes is about $1 \mathrm{~ns}$ and the fall time is less than $0.1 \mu \mathrm{s}$. Thus, the GaN/AlGaN p-i-n photodiode response to UV radiation is much faster than the response requirements needed for UV FPA applications. FPA camera frame rates of from $15-240 \mathrm{~Hz}$ are generally employed, corresponding to pixel integration times from about $1 \mathrm{~ms}$ up to $65 \mathrm{~ms}$ per frame, depending on the camera frame rate and the available UV photon flux. The fast turn-on of the GaN/AlGaN p-i-n photodiodes implies that very short UV bursts of radiation -such as might be expected from shell fire, for example -can also easily be detected by the nitride detectors and detector arrays.

Figure 7 shows a comparison of the digital imagery obtained using the $128 \times 128$ UV FPA with that of imagery obtained earlier using a 32x32 UV FPA [15]. The increased resolution of the $128 \times 128$ is clearly seen upon comparison of the two types of alphanumeric images shown in the figure. 
Figure 8 shows additional comparisons of images of geometric figures obtained using the $32 \times 32$ and $128 \times 128$ UV FPAs, respectively. It is seen that the $128 \times 128$ imager produces much clearer images of all of the geometric figures, particularly for the circles and triangles.

Figure 9 shows both a visible image of a tungsteninert-gas (TIG) arc welder and the corresponding UV emission (320-365 $\mathrm{nm}$ ) from the arc welder that was obtained using the $128 \times 128$ FPA. The imagery was conducted in the physics machine shop at NCSU. In order to obtain the UV image shown, it was necessary to use a neutral density \#4 filter in front of the UV camera to reduce the UV emission intensity from the arc welder and thus avoid pixel saturation. Figure 10 shows similar UV imagery (320-365 nm) obtained from an oxy-acetylene torch.

The UV images shown in Figures 9 and 10 correspond to single frames of UV movies of the TIG welder and torch recorded at $15 \mathrm{~Hz}$. Movies of both of these UV sources were obtained using frame rates up to 240 $\mathrm{Hz}$.

\section{Summary and Conclusions.}

Visible-blind photodiode structures have been successfully synthesized using MOVPE. The devices were fabricated into test structures and observed to have high detectivity and time response properties, which make them suitable for UV imaging applications. These device structures were fabricated into $128 \times 128$ arrays of detectors using standard photolithography, dry etching, and metallization processes. The imaging arrays were hybridized to a Si ROIC using In bump bonds. The hybridized UV imager was operated at room temperature over a range of frame rates from $15-240 \mathrm{~Hz}$. The visible-blind imager was used to observe a variety of ultraviolet scenes. Digital images were acquired and stored using computer-controlled hardware. A selection of the observed images is presented in this work.

The UV camera demonstration based on a $128 \times 128$ array of GaN/AlGaN p-i-n photodiodes that is reported herein represents another important advancement in optoelectronic devices based on III-V nitride materials. The format of these new UV-specific imagers (128x 128 pixels) is compatible with corresponding imagers in the near-IR and far-IR spectral regions. Thus, multispectral imagery ranging from the UV to the far IR is now possible with a suitable integration of this new UV camera device.

Based on our work to date, we believe that it will be possible to make $1024 \times 1024$ pixel imagers within the next year or two. Applications for these imagers include biological agent detection, missile and shellfire detec- tion, atmospheric ozone-level detection, welding imagery, and flame sensing. In addition, large-format UV staring FPAs based on nitride photodiodes may play an important role in obtaining UV images of the stars and other astronomical objects of importance in understanding the creation and evolution of the universe.

\section{ACKNOWLEDGMENTS}

The work at NCSU is being supported by Army Research Office grants DAAH04-95-1-0627 and DAAD19-99-0113 and by Defense Advanced Research Projects Agency grant DAAD19-99-0010 under the Solar Blind Detector Program directed by Dr. E. Martinez. Honeywell would like to acknowledge the support and encouragement of Dr. Paul Schreiber at the Air Force Research Laboratories, Wright-Patterson Air Force Base, Ohio.

\section{REFERENCES}

[1] Shuji Nakamura, Takashi Mukai, Masayuki Senoh , Appl. Phys. Lett. 64, 1687-1689 (1994).

[2] Shuji Nakamura, Masayuki Senoh, Naruhito Iwasa, Shin-ichi Nagahama, Appl. Phys. Lett. 67, 1868-1870 (1995).

[3] S Nakamura, M Senoh, S Nagahama, N Iwasa, T Yamada, T Matsushita, Y Sugimoto, H Kiyoku, Appl. Phys. Lett. 70, 1417-1419 (1997).

[4] Shuji Nakamura, Gerhard Fasol, The Blue Laser Diode - GaN based Light Emitters and Lasers , (Springer-Verlag, Heidelberg, 1997), .

[5] F. A. Ponce, MRS Bull. 22, 51-57 (1997).

[6] J Edmond, HS Kong, M Leonard, G Bulman, G Negley, Inst. Phys. Conf. Ser. 142, 991 (1996).

[7] I. Akasaki, S. Sota, H. Sakai, T. Tanaka, M. Koike, H. Amano, Electron. Lett. 32, 1105-1106 (1996).

[8] G.E. Bulman, K. Doverspike, S.T. Sheppard, T.W. Weeks, H.S. Kong, H.M. Dieringer, J.A. Edmond, J.D. Brown, J.T. Swindell, J.F. Schetzina, Electron. Lett. 33, 15561557 (1997).

[9] M.P. Mack, A. Abare, M. Aizcorbe, Peter Kozodoy, S. Keller, U. K. Mishra, L. Coldren, Steven DenBaars, MRS Internet J. Nitride Semicond. Res. 2, 41 (1997).

[10] S Nakamura, M Senoh, S Nagahama, N Iwasa, T Yamada, T Matsushita, H Kiyoku, Y Sugimoto, T Kozaki, H Umemoto, M Sano, K Chocho, Appl. Phys. Lett. 72, 211213 (1998).

[11] Dennis K. Wickenden, Zhenchun Huang, D. Brent Mott, Peter K. Shu, Johns Hopkins APL Tech. Dig. 18, 217 (1997).

[12] Wei Yang, Thomas Novova, Subash Krishnankutty, Robert Torreano, Scott McPherson, Holly Marsh, Appl. Phys. Lett. 73, 1086 (1998).

[13] Hadis Morkoc, Nav. Res. Rev. 51, 26 (1999).

[14] T. Huang, D. B. Mott, A. La, Proc. SPIE 3765, 254 (1999).

[15] J.D. Brown, Zhonghai Yu, J. Matthews, S. Harney, J. Boney, J.F. Schetzina, J.D. Benson, K.W. Dang, C. Terrill, 
Thomas Nohava, Wei Yang, Subash Krishnankutty, MRS Internet J. Nitride Semicond. Res. 4, 9 (1999).

[16] J. D. Brown, J. Matthews, S. Harney, J. C. Boney, J.

F. Schetzina, J. D. Benson, K. V. Dang, Thomas Nohava,
Wei Yang, Subash Krishnankutty, MRS Internet J. Nitride Semicond. Res. 5S1, W1.9 (2000). 


\section{FIGURES}

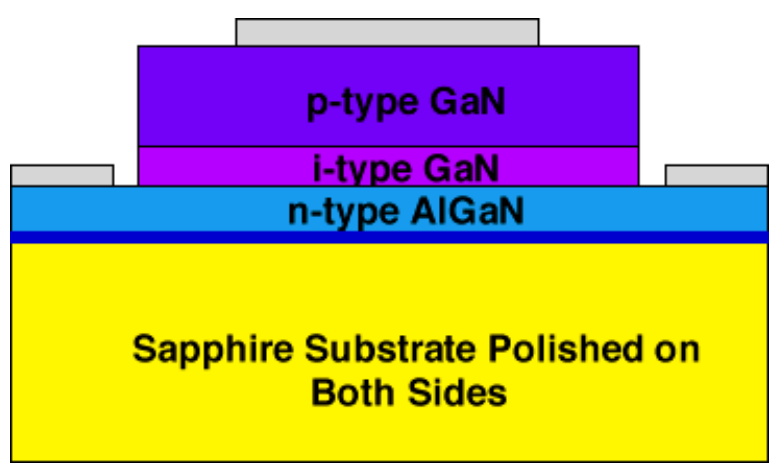

Figure 1. Schematic of GaN/AlGaN p-i-n photodiode structure. 


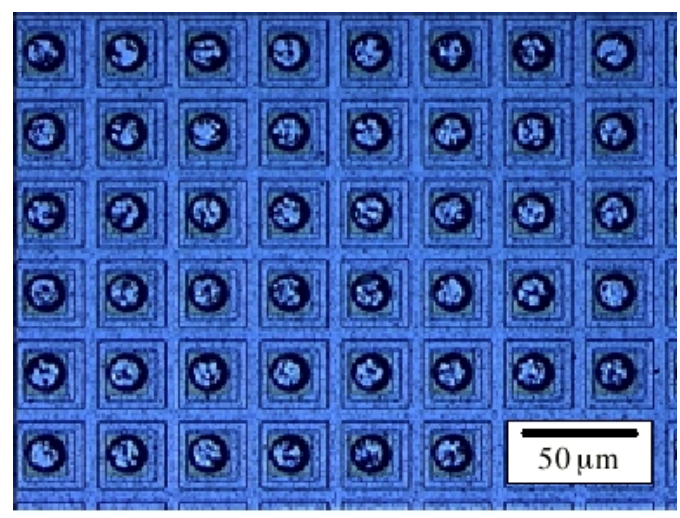

\section{8x128 Photodiode Array}

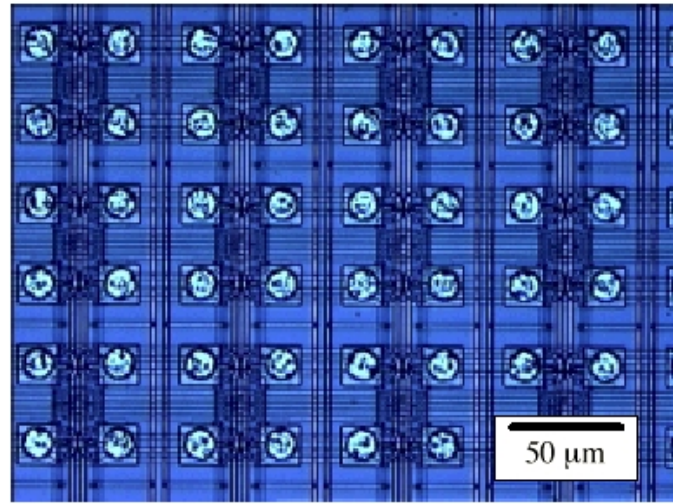

$128 \times 128$ Silicon ROIC

Figure 2. Nomarski photograph of photodiode array and Si ROIC with deposited In bumps. 


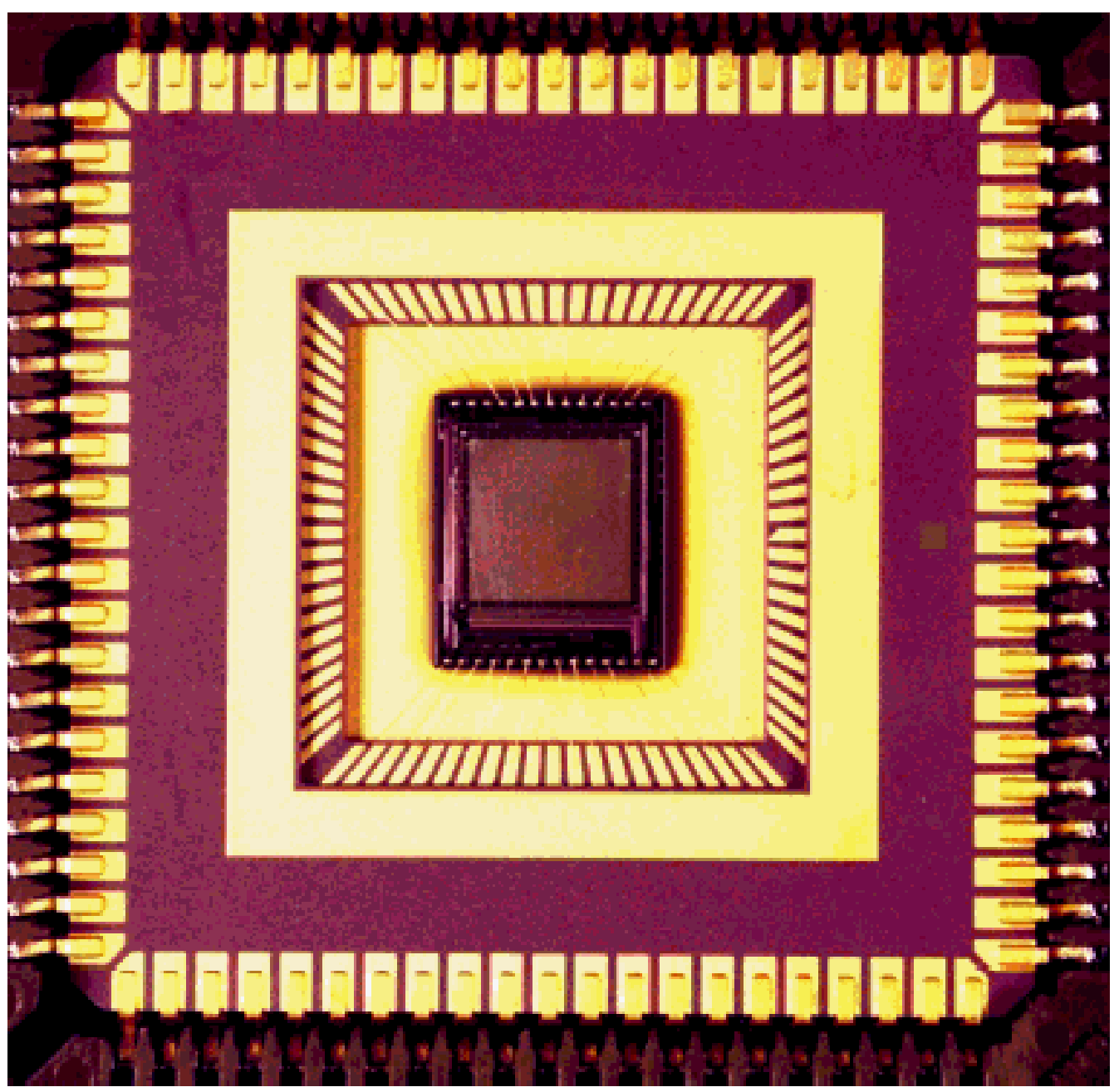

Figure 3. Hybridized focal plane array imager mounted and wirebonded to 84 pin leadless chip carrier. 

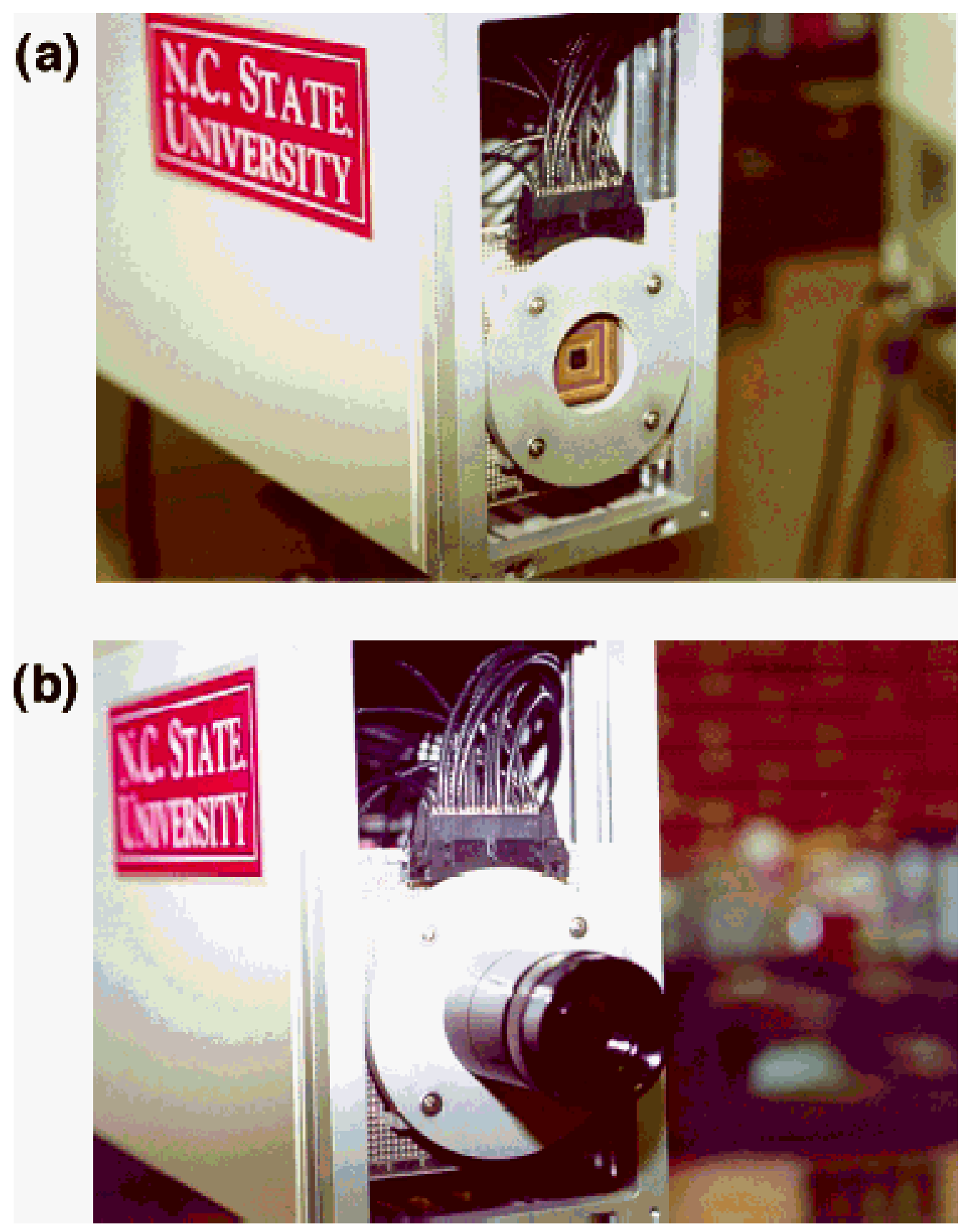

Figure 4. (a) UV FPA chip assembly mounted in LCC socket in UV camera head; (b) Camera head with quartz UV optics installed. 


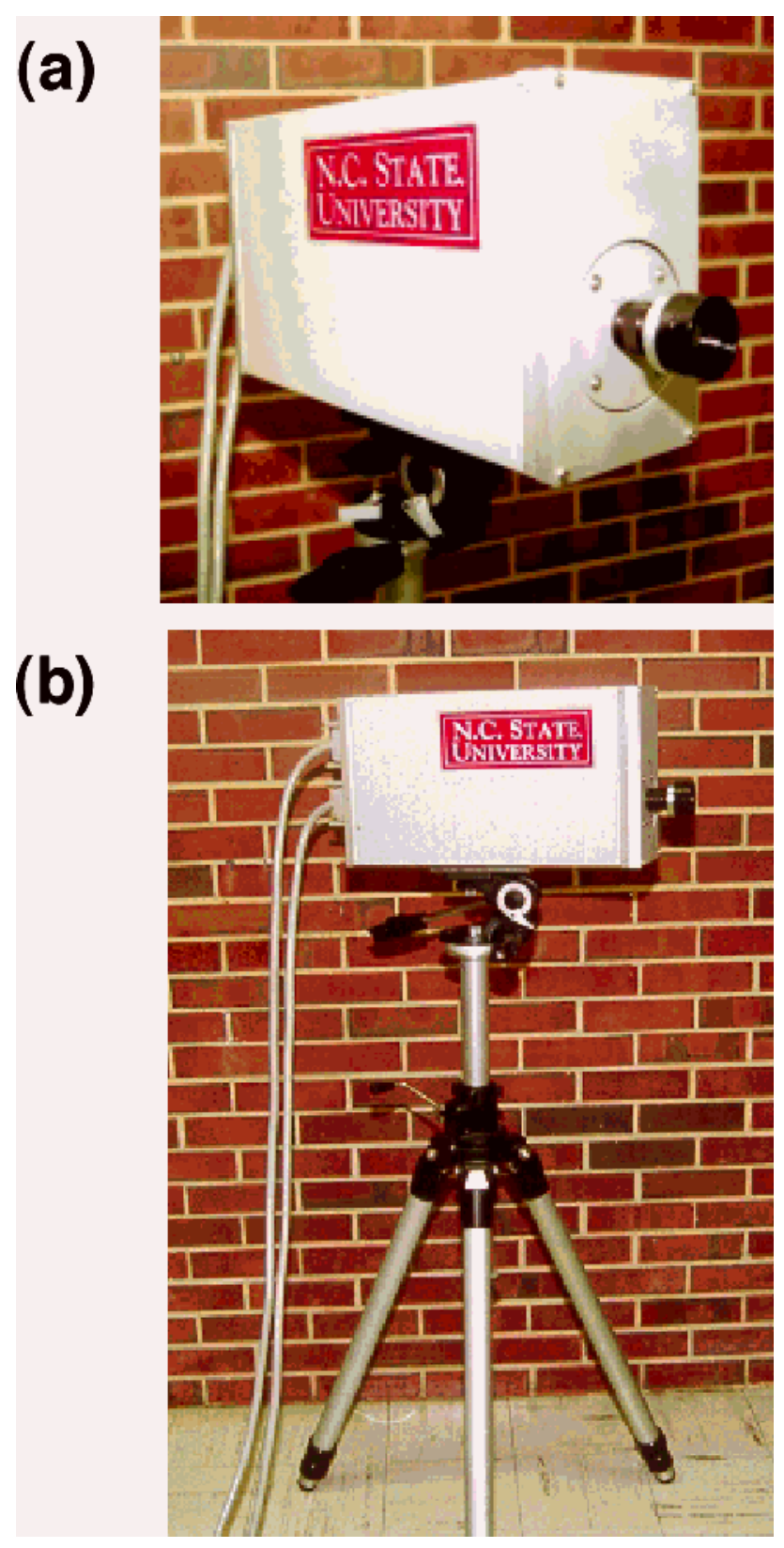

Figure 5. Two views of NCSU UV camera. 


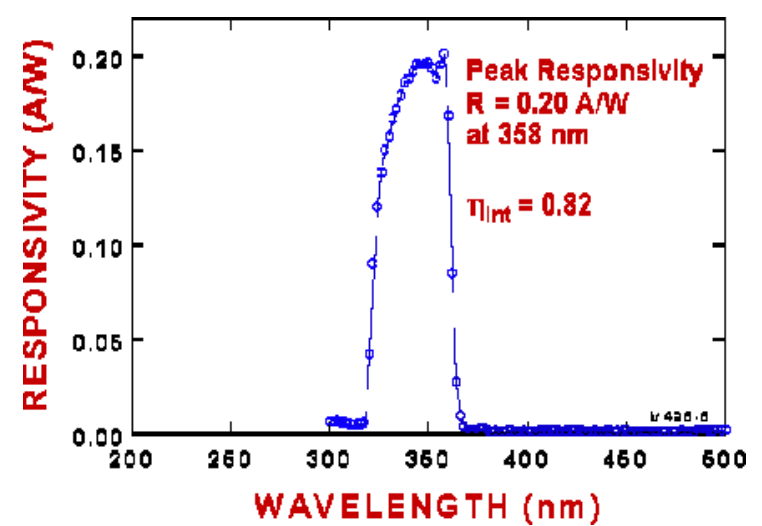

Figure 6. Spectral responsivity of discrete $(200 \mu \mathrm{m} \times 200 \mu \mathrm{m})$ $\mathrm{GaN} / \mathrm{AlGaN}$ heterostructure photodiode operating in the photovoltaic mode (zero bias).
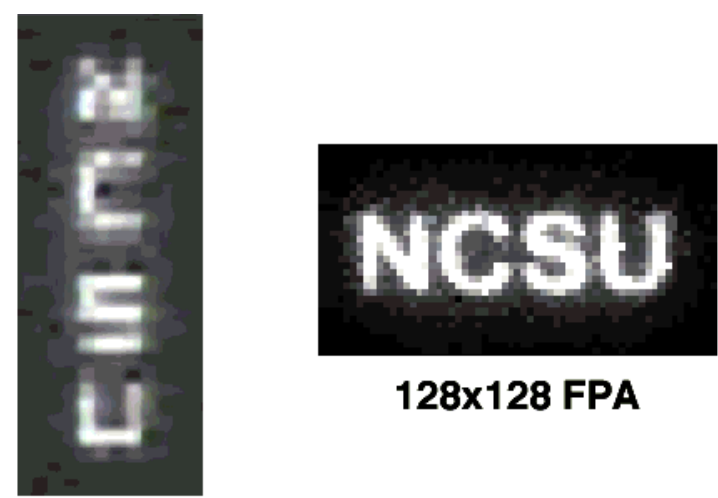

$128 \times 128$ FPA

\section{2x32 FPA}

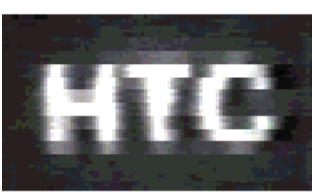

$32 \times 32$ FPA

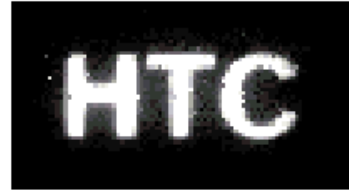

$128 \times 128$ FPA
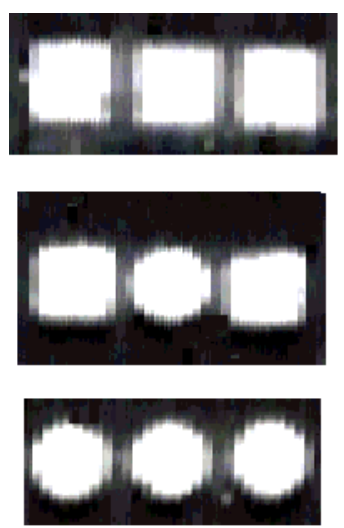

$32 \times 32$ FPA
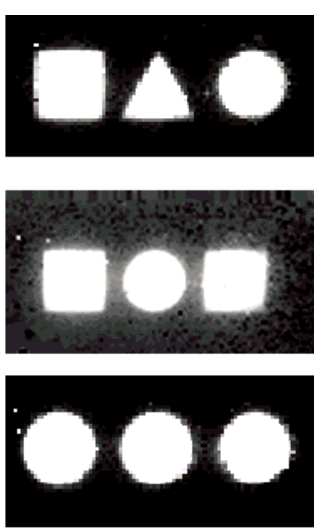

$128 \times 128$ FPA
Figure 8. Comparison of geometric images obtained using a $32 \times 32$ UV FPA versus a 128x128 UV FPA.

Figure 7. Comparison of alpha-numeric images obtained using a 32x32 UV FPA versus a 128x128 UV FPA. 

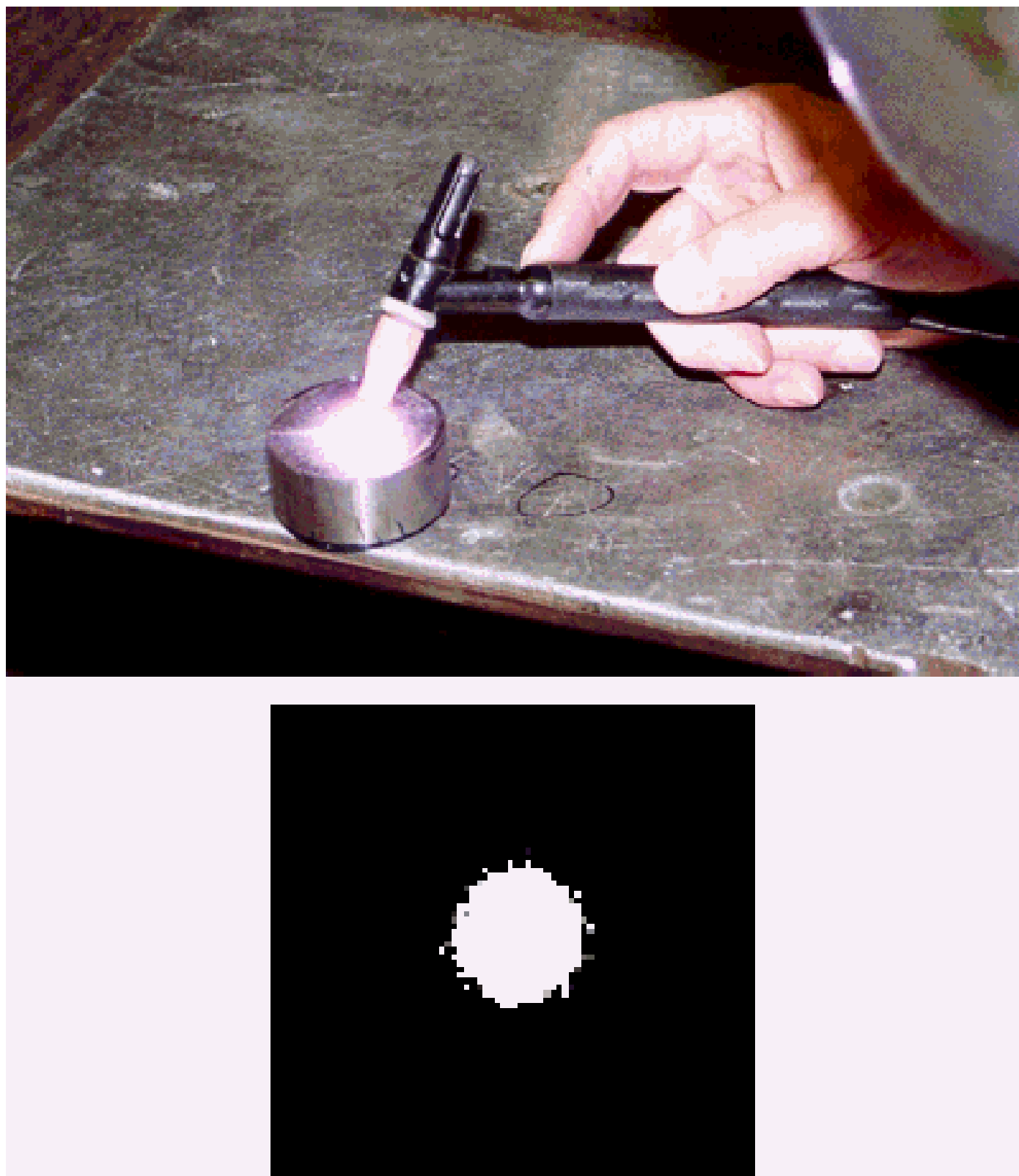

Figure 9. Visible (top) and UV (320-365 nm) visible-blind image of TIG arc welder. 

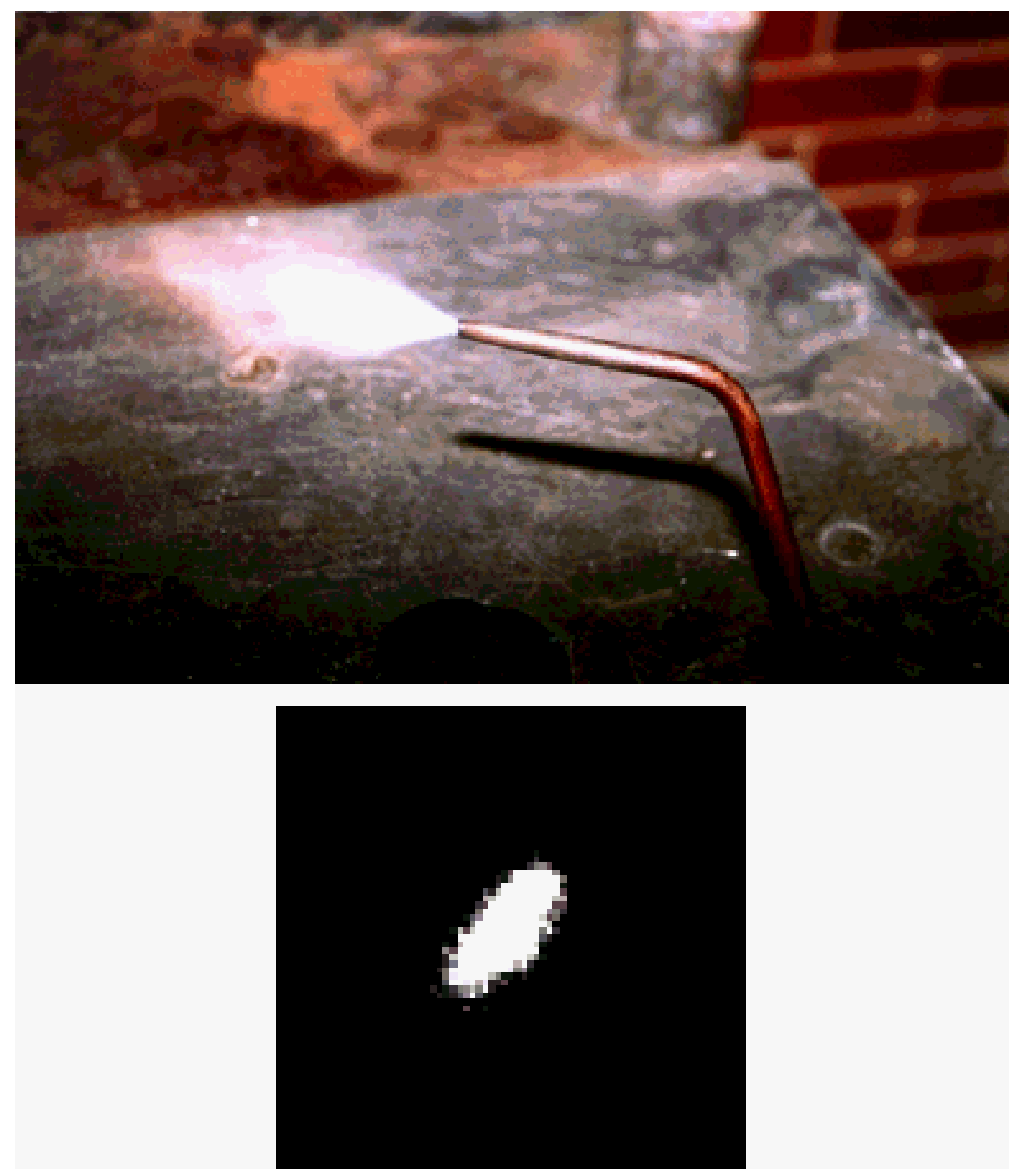

Figure 10. Visible (top) and UV (320-365 nm) visible-blind image of oxy-acetylene torch. 